\title{
LA STYLISTIQUE DE M. CHARLES BALLY
}

By OtTo MÜLleR

$\mathbf{L}^{\mathrm{E}}$

E Traité de stylistique française de M. Charles BaLly a paru récemment en deuxième édition. Le moment semble venu de rappeler très rapidement ce que l'auteur entend par stylistique et d'examiner les avantages que l'enseignement des langues modernes peut retirer d'une méthode générale d'investigation qui ne se confond pas avec la stylistique, tout en lui étant nécessaire.

Les écrits de M. Bally sur la stylistique sont les suivants: Précis de stylistique, Genève 1905; Traité de stylistique, Heidelberg 1909, deuxième édition 1920, en deux volumes; L'étude systématique des moyens d'expression, Genève 1910; La stylistique française de 1905 d la fin de 1909, dans le Romanischer Jahresbericht de K. Vollmöller, vol. XI, p. 189 s.; Stylistique et linguistique gênêrale, dans le Archiv für das Studium der neueren Sprachen, vol. CXXVIII, p. 87-126; La stylistique et l'enseignement secondaire, St-Blaise 1911; Le style indirect libre en français, dans la Germanisch-Romanische Monatsschrift de 1912, p. 549 s. et 597 s.; Le langage et la vie, Genève 1913; Figures de pensée et formes linguistiques, dans la Germanisch-Romanische Monatsschrift de 1914, p. 405 s. et 456 s.; Stylistique génerale et stylistique française, dans le Romanischer Jahresbericht de K. Vollmöller, vol. XIII, p. 190 s.; Ferdinand de Saussure et l'état actuel des études linguistiques, Genève 1913; Impressionnisme et Grammaire, dans les Melanges d'histoire littéraire et de philologie offerts d $M$. Bernard Bouvier, p. 261 s., Genève 1920; L'enseignement de la langue maternelle et la formation de l'esprit, dans Le Producteur, vol. IV, p. 354 s., Paris 1921.

Demandons-nous d'abord ce que M. Bally comprend sous le nom de stylistique. Lorsqu'on entend ce mot, on pense immédiatement au style d'un écrivain. Or la stylistique telle que la conçoit M. Bally n'a rien à voir avec le style, clle ne gravite pas autour de l'expression littéraire. Elle étudie le langage affectif naturel et tient sa place entre plusieurs disciplines fort différentes. D'abord elle relève de la linguistique statique, et s'attache aux valeurs expressives de la langue, où qu'elles se trouvent, dans les mots et dans la grammaire. Mais avant d'édifier un système, M. Bally expose sa méthode d'investigation dans une première 
partie du Traité de Stylistique. Ce travail préparatoire assez long a contribué à donner au mot stylistique un sens trop large. Cette partie du Traitê est néanmoins presque la plus intéressante. Nous y trouvons le développement des deux principes sans lesquels toute recherche stylistique est impossible: 1) Les méthodes traditionnelles d'enseignement font de l'étude d'une langue un travail trop automatique, trop analytique et historique; 2) l'observation doit porter sur les rapports existant entre les signes linguistiques et les relations qui unissent la parole à la pensée, ce qui est incompatible avec l'étude historique. ${ }^{1}$

Le dualisme de la linguistique statique et de la linguistique historique a été accentué par Ferdinand de Saussure dans son Cours de linguistique générale (Paris, Payot 1916). Mais c'est M. Bally qui l'a reconnu d'abord et qui en a montré la portée pratique;" il dit ailleurs: "lorsque ce principe de la dualité des deux linguistiques-universellement violé aujourd'hui-sera reconnu et appliqué, il transformera profondément nos méthodes."’3

La stylistique s'emboîte donc dans la linguistique statique, qui étudie la langue par l'observation des oppositions entre les éléments de la langue.

La délimitation des faits de langue, conforme à leur signification, est une autre grande tâche de la linguistique statique. Il s'ensuit que la délimitation, comme l'identification des significations, est une préparation indispensable à toute étude stylistique, bien que ces opérations ne se confondent pas avec la discipline spéciale appeléc stylistique. La méthode appliquée surtout dans les gymnases classiques de l'Europe, qui consiste à confondre l'unité avec le mot tel qu'il est délimité par l'écriture, n'a pas encore été abandonnéc. On est toujours tenté de procéder trop analytiquement et de disséquer une phrase en des éléments qui ne sont tels que sur le papier. Prenons par exemple l'expression avoir maille a partir (avec quelqu'un), citée par M. Bally au Traité p. 56. Aucun de ces mots n'a conservé sa valeur propre étymologique, mais aucun n'a pris, isolément, une signification différente; ils font corps avec l'unité de pensée que la locution est chargée d'exprimer. On sait qu'elle signifie "être en désaccord, avoir un différend avec

1 Traite, I, p. 2.

${ }^{2}$ Traile, p. $21 \mathrm{~s}$.

${ }^{3} \mathrm{~F}$. de Saussure et l'étal actuel des êtudes linguistiqucs, p. 16. 
quelqu'un." C'est en partant de l'unité correspondant à une idée déterminée qu'une méthode psychologique devra expliquer le véritable rapport entre la pensée et la parole. Ce n'est donc pas l'étymologie de ces mots qui en révèlera la signification réelle, actuelle, ni la valcur expressive qu'elle a aujourd'hui, car les Français ne savent plus que maille signifiait "pièce de monnaie" et partir "partager"; cette explication n'a de valeur vraie que pour les philologues; une périphrase donnant la signification exacte de la locution en question a une valeur pratique bien autrement considérable.

Dans certains cas il peut même arriver que l'étymologie produise une idée fausse, ainsi quand nous disons que décamper dans le sens de "s'éloigner rapidement" vient de camp. Dans la langue usuelle personne ne pensera à ce dernier terme. Les élèves des gymnases d'Europe et les "Undergraduates" de nos collèges ne sauraient faire de l'étymologie une étude systématique. Il suffit qu'on leur donne de temps en temps un aperçu du cours de l'évolution de la langue étudiée. Il importe avant tout de leur faire saisir la signification actuelle des termes d'une langue étrangère. On est souvent frappé chez l'étranger d'un certain instinct à décomposer les unités plus que ne le font les sujets qui parlent leur langue maternelle; cette décomposition ne rend que plus difficile l'assimilation de faits linguistiques nouveaux. ${ }^{4}$ L'étranger cherchera, en considérant seulement la forme extérieure des mots, à relier entre cux des groupes qui ne sont plus dans un rapport étymologique étroit. Par là il risque de leur attribuer une fausse signification, alors qu'il importerait de connaitre le sens actuel de ces mots.

Si d'une part $M$. Bally préconise l'étude des faits d'expression par la méthode descriptive, il constate d'autre part qu'il est très difficile de se passer entièrement, surtout au commencement de l'étude d'une langue, de l'aide mnémotechnique que nous apporte le groupement des mots par familles. C'est pour cette raison qu'il nous invite à un compromis. Mais ne relevons que les rapports étymologiques frappants, qui sont encore sentis à l'heure actuelle. Dès qu'on aura fait les premiers pas dans l'étude d'une langue, il faudra se passer de cette méthode, pour la remplacer par le groupement rationnel des faits d'expression.

${ }^{4}$ Truilé, J, p. $32 \mathrm{~s}$. 
Après la délimitation vient le problème de l'identification des faits d'expression. Du reste les deux questions se posent en même temps. Identifier une expression telle que "avoir maille à partir" c'est trouver l'idée simple qu'elle exprime, en ce cas l'idée de désaccord, querelle etc. Cette longue préparation permet dès lors à M. Bally de tracer les limites de son champ d'étude: la stylistique ou l'étude des aspects affectifs et expressifs de la langue de tout le monde.

Prenons un exemple, soit la locution décampez au plus vite. Nous déterminerons d'abord l'idée simple renfermée dans le verbe: décamper signifie "se retirer," "fuir," "se sauver." Mais aucune de ces expressions ne correspond absolument à celle qu'il s'agit d'expliquer. Comment et par où se distingue-t-elle des autres? En consultant Littré, nous y lirons que decamper = se retirer précipitamment. Mais décamper contient à côté de son sens intellectuel encore deséléments affectifs que l'on ne saurait négliger. C'est ainsi que nous y sentons exprimé un éloignement forcé, puis l'ordre est exprimé d'une façon familière. Nous traduirions en anglais la locution en question par "hurry up and get out." Nous sommes transportés du coup dans un milieu social bien déterminé et nous nous représentons par exemple un maitre qui s'adresserait ainsi à des écoliers qui lui auraient joué quelque mauvais tour.

Toute expression renferme donc à côté de certains aspects affectifs naturels aussi des caractères sociaux qui agissent à leur tour affectivement. L'étude de ces nuances expressives avait été assez négligée jusqu'ici. On peut citer dans cet ordre d'idées entre autres l'étude de Tobler sur la locution par exemple. Mais ce sont les livres de M. Bally qui font des nuances expressives une étude systématique et qui one créé une science étudiant les moyens d'expression d'une langue d'après leur contenu affectif. Il est vrai que les considérations de Groeber sur la syntaxe affective ont précédé les ouvrages de M. Bally, mais ce dernier nous dit qu'il n'en avait pas pris connaissance lorsqu'il composa son Traité de Stylistique. ${ }^{5}$

On a reproché à l'auteur du Traité son emploi du terme stylistique. On l'a blâmé d'avoir reculé devant la création d'un terme nouveau; il convient lui-même que c'est une faiblesse, ${ }^{6}$ et que ce terme manque de précision, parce qu'il désigne un ensemble mal

- Jahresbericht de K. Vollmöller XI, 194 en note.

- Traile, avant-propos p. IX. 
défini de recherches, limitrophes de la grammaire, de l'art d'écrire et de la littérature. ${ }^{7}$ La stylistique était jusqu'ici l'étude du style, et toutes les définitions, fussent-elles exprimées le plus généralement possible, étaient toujours orientées vers l'étude d'une langue vue sous un point de vue artistique. Littré, dans le Dictionnaire de la langue française définit la stylistique "la théorie du style." Darmesteter et Hatzfeld ne connaissent meme pas ce mot. Le Nouveau Larousse explique: "La stylistique tient le milieu entre la grammaire et la rhétorique, elle donne des règles sur le choix des expressions, sur l'emploi des parties du discours, sur la construction des périodes, et met en relief les caractères essentiels d'une langue donnée." Mais au lieu de considérer l'aspect artistique, M. Bally fait intervenir le sentiment pur et simple, et au lieu d'étudier la langue littéraire, il ne s'attache qu'à la langue parlée. M. W. Küchler a fait observer que M. Bally fait intervenir ici un changement du sens de ce mots et il ne reste qu'à savoir si ce sera son opinion qui prévaudra à l'avenir. Presque tous les critiques qui se sont occupés des livres de M. Bally lui ont fait des reproches à ce sujet. M. Wilmotte écrit à propos du Précis de Stylistique: "Je ne le querellerai que sur son titre et sa définition de la stylistique qui bouscule des habitudes reçues sans profit pour personne. Un traité de la rédaction ou de l'élocution française n'est pas une stylistique, et c'est introduire une fâcheuse terminologie allemande ( $?$ ) que d'appeler de ce dernier nom un traité, d'où le souci d'un style littéraire est absent. ${ }^{9}$ M. W. Küchler s'exprime dans le meme ordre d'idées, ${ }^{10}$ ainsi que $M$. Th. Kalepsky ${ }^{11}$ qui dit: "Wenn ich auch weit davon entfernt bin, ihm das Recht zum Gebrauch des Wortes Stilistik in der von ihm "genau präzisierten" Bedeutung streitig zu machen, so kann ich doch nicht umhin, in seinem Interesse, wie in dem mancher Käufer des Buches zu bedauern, dass er nicht wenigstens für den Titel eine minder leicht zu missdeutende Bezeichnung gewählt hat."

La stylistique n'est pas non plus, comme l'ont cru dans la suite certaines personnes, l'étude des expressions inusitées, singulières,

'Romanischer Jahresbericht XI, 189.

- Archiv CXXII, 196.

- Zeitschrift filr franzobsische Sprache und Literatur XXX, 182.

${ }^{10}$ Archiv l.c.

" $Z$ f $S L \mathrm{XXXVI,} \mathrm{154-158.}$ 
l'étude, par exemple, de l'argot. Il s'agit, au contraire, pour elle d'étudier les formes les plus simples de la langue de tout le monde, celles que nous employons automatiquement, sans y prendre garde, pour produire un effet affectif. Ainsi pour prendre un exemple de l'anglais, elle se demandera pourquoi et en quelles circonstances on dira plutôt "quite well" que "very well." Elle n'attachera aucune importance aux formes individuelles, qui naissent au cours de la conversation et disparaissent aussi rapidement qu'elles sont nées.

Dans d'autres publications M. Bally a étudié les différents sens qu'a reçus le mot de stylistique. ${ }^{12}$ Ces distinctions lui permettent de mieux préciser ce qu'il entend, lui, par ce terme. Il distingue 1. la stylistique externe, 2. la stylistique interne, 3 . la stylistique littéraire, et c'est la seconde qui, comme nous le verrons, correspond à la définition de M. Bally.

La stylistique externe cherche à découvrir les caractères fondamentaux d'un idiome en les comparant avec ceux d'un autre idiome. C'est la méthode suivie en Allemagne depuis la Stylistique latine de Naegelsbach; elle opère par comparaison et par traduction, c'est la méthode philologique par excellence. Sans être dépourvue de valeur, elle ne permet que de saisir les manifestat ons de notre pensée, elle n'en considère que les symboles extérieurs. Le fait que ces symboles sont examinés attentivement et l'effort qu'en nécessite l'interprétation auront pour résultat que les caractères de cette langue seront vus sous un angle intellectuel et notre être n'est pas tout intellect. Ces travaux reposent en outre sur la grammaire et celle-ci étant restée formelle et scolastique, les résultats seront stériles. Nous en sommes pour la grammaire au point où les Grecs l'avaient amenée, nous procédons comme eux selon des règles empiriques. ${ }^{13}$ Sans écarter tout à fait cette méthode (qu'il propose d'appeler la stylistique externe), M. Bally n'adopte cependant pas la définition de Ries qui voit en la stylistique d'une langue l'étude des caractères de cette langue;"14 ces caractères, linguistiques dans leur nature, reflètent les caractères psychiques de la collectivité qui parle cet idiome. Mais nous pouvons être frappés par une particularité linguistique qu'un étranger ne remarquerait pas et vice-versa, ce qui le frapperait

12 Jahresbericht de K. Vollmöller, XIII $191 \mathrm{~s}$.

${ }^{13}$ Voir Trailé 1,27 et Archiv CXXVIII, 93.

"Was ist Syntax? p. 127. 
nous laisserait indifférents. En dernier lieu il peut arriver que nous soyons impressionnés tous deux, mais d'une façon différente. La méthode d'observation que $M$. Bally oppose à celle-ci, et que nous avons caractérisée plus haut, consiste à comparer les principaux types expressifs de la même langue. Il lui réserve le nom de stylistique interne. Cette méthode sera psychologique ou elle ne sera pas, dit-il quelque part. ${ }^{15}$ Elle "étudie la langue comme système de faits expressifs et de réactions impressives tels qu'ils se montrent à la réflexion intérieure ou introspection." 1 E Elle considérera les modifications subies par la langue organisée au contact de la vie réelle. Si nous nous reportons à la page 16 de Le langage et la vie nous y apprenons ce que l'auteur entend par vic en matière de langage. Il s'agit de la conscience et de la volonté de vivre, du sens vital que nous sentons en nous-mêmes. La vie n'étant pas actionnée par des idées pures, le langage qui en est l'expression ne saurait être une création logique. Par opposition à la langue organisée, qui est intellectuelle et logique, surgit un langage affectif, qui est comme le principe vital de la langue. Il agit sans cesse, car au contact de la vie, les idées les plus objectives en apparence s'imprègnent d'affectivité et deviennent des jugements de valeur. ${ }^{17}$ Ceux-ci s'émoussant à leur tour, rentrent dans la langue usuelle. L'action des sujets parlants sur la langue dite intellectuelle ou normale est comparéc par $M$. Bally à un siège que la parole fait subir à la langue. Les individus luttent contre la langue organisée, parce qu'elle ne les satisfait presque jamais complètement. Les créations affectives entourent en quelque sorte comme d'une zone périphérique la langue normale. Une partie de ces produits de l'affectivité pénètre dans la langue proprement dite et vient grossir le stock des expressions qu'étudie la stylistique.

M. Bally applique à ces transformations du langage spontané l'image de la toile de Pénélope qui se fait et se défait sans cesse parce que l'intelligence et la sensibilité y travaillent, mais non pas toutes deux de la même façon. Il peut alors se produire qu'un même mot ait un sens purement intellectuel et un sens subjectif et affectif. ${ }^{18}$ M. Bally cite l'exemple de dramatique. Dans "l'art

\footnotetext{
${ }^{16}$ Ferdinand de Saussure et l'étal actucl des études linguisliques, p. 22.

16 Archiv CXXVIII, 96.

17 Le langage el la vie, p. 24.

${ }^{18}$ Le langage el la vie, p. 25.
} 
dramatique" aucune nuance d'émotion, tandis que "un incident dramatique" exprime une valeur subjective. On peut donc dire que presque chaque mot renferme des éléments qui s'adressent au pur entendement et ont un sens objectif, tandis que d'autres s'adressent à nos émotions et ont un sens subjectif. Ces éléments subjectifs ou impressifs seront absents par exemple des termes scientifiques, alors que les mots du langage populaire en seront tout imprégnés. En d'autres termes, tout revient en stylistique à savoir si un fait linguistique s'adresse à la sensibilité et à l'imagination ou à l'entendement pur et simple. M. Bally va jusqu'à dire "Nous parlons donc simultanément deux langues," désigner par les mots objectif et subjectif ou encore logique et affectif. La première exprime des idees pures, la seconde les présente sous forme de sentiments. Ayant pour objet la langue affective, la stylistique interne doit étudier avec autant d'attention la langue intellectuelle, puisque l'expressivité résulte du contraste entre ce qui est affectif et ce qui ne l'est pas. M. Bally nous dit le but qu'il assigne à cette stylistique: "Je considère comme la tâche de la stylistique interne de rechercher quels sont les types expressifs qui, dans une période donnée, ont servi ou servent à rendre les mouvements de la pensée et du sentiment des sujets parlants, et d'étudier les effets produits spontanément chez les sujets entendants par l'emploi de ces types expressifs."

Il reste à examiner ce qu'on a appelé la stylistique littéraire, c'est-à-dire l'étude des procédés de langage en tant qu'ils acquièrent une valeur esthétique lorsqu'ils sont employés par un écrivain, ce qui représente l'ancienne acception du terme. Nous avons vu que dans ce sens, la stylistique ne se confond pas avec celle qu'étudie M. Bally. L'expression littéraire ou style apparalt dans le système de ce dernier comme une "recréation à la fois consciente et esthétique du langage de la vie." 21 Partant des créations individuelles nous voyons que les effets littéraires sont contenus en germe dans la langue de tout le monde. L'artiste seul les emploie en vue de l'émotion littéraire, dans la vie ordinaire au contraire les sujets s'en servent essentiellement comme moyens d'expression et d'action, sans aucune visée esthétique. La langue littéraire est

${ }^{19}$ Précis de slylistique, p. 130.

${ }^{20}$ Archiv CXXVIII, 94.

"Romanischer Jahresbericht XIII, 193. 
donc sortie du langage spontané. Ce n'est pas tout: M. Bally distingue encore avec soin la langue littéraire du style. ${ }^{22}$ La langue littéraire est constituée par la fusion des styles, par tous les éléments littéraires accumulés à travers les générations. Elle a une valeur sociale, celui qui l'emploie montrant par là même son éducation supérieure. Pour la linguistique elle est une langue spéciale, comme le langage scientifique ou même l'argot. Le style au contraire est la marque distinctive d'un esprit, il dénote une création personnelle. Tout texte littéraire porte, à des degrés divers, la marque personnelle de l'auteur. Le danger des recherches stylistiques est qu'elles aboutissent souvent à l'effacement des caractères personncls d'un autcur par la traduction. Aussi M. Bally ne veut-il rien savoir de la traduction, même pour l'étude des mots. ${ }^{23}$ On devrait s'habituer à les expliquer au lieu de les traduire. Nous pensons aussi que ce genre d'exercice devrait avoir une place plus importante dans l'enseignement du français aux Etats-Unis. Rien n'est plus utile que de forger une définition et de la contrôler avec celle que donne un bon dictionnaire explicatif. En outre les définitions sont des spécimens typiques de langage objectif, une définition étant formée d'expressions dépouillées de leur contenu affectif. Il est évident que la stylistique et l'étude des formes littéraires se tiennent de près, car celles-ci sont aussi fondées sur les faits de sensibilité; toute nuance littéraire doit en effet produire un minimum d'émotion. Mais il importe d'étudier les faits de la sensibilité là où on peut les saisir le plus facilement dans toute leur pureté, c'est-à-dire dans le langage ordinaire.

La stylistique, avons-nous dit, n'est pas une science historique. C'est là un point capital des idées de M. Bally. Qui dit histoire dit évolution; mais le sujet parlant n'a jamais conscience d'une évolution lorsqu'il parle sa langue maternelle et c'est parce que les signes linguistiques coexistent et s'opposent dans le même temps dans son cerveau, que ces signes ont une signification, et que les uns sont expressifs et que les autres ne le sont pas. Les sujets parlants croient que la langue qu'ils parlent a toujours été telle. C'est là une constatation si évidente qu'il paraît surprenant qu'on ait pu dire qu'Adolphe Tobler s'est occupé de l'ancien français plutôt que du français moderne "weil gerade das Altfran-

${ }^{22}$ Le langage et la vie, p. 50.

${ }^{23}$ Precis, p. 36. 
zösische als eine Zeit des Chaos, aus dem sich etwas Neues entwickeln wollte . . f für den Sprachforscher besonders lehrreich ist."24 Mais les auteurs de l'ancienne France avaient, tout comme les écrivains d'aujourd'hui, le sentiment non pas de l'évolution, mais de l'état fixe de leur langue, de la langue parlée qu'ils transposaient en vue d'un effet littéraire.

M. Bally dit quelque part très clairement le but pratique qu'il voudrait voir atteint: "A côté de l'étude historique des faits de langage, la linguistique doit faire une place à la science de l'expression et à l'étude des élats de langage" . . . les langues vivantes "s'expliquent tout autant, si ce n'est mieux, par leur réalité actuelle que par leur passé lointain." ${ }_{25}$

Et maintenant comment un étranger doit-il s'y prendre pour faire des études stylistiques? Le point de départ, nous l'avons $\mathrm{vu}$, est l'observation de la langue parlée, la langue écrite n'en étant qu'unc sorte de transposition. Il s'agit de se pourvoir d' "impressions linguistiques," c'est-à-dire de collectionner telles quelles des locutions "impressives." Même si l'on ne devait pas tout comprendre de prime abord, l'expérience aidant, on s'y retrouvera, à force d'entendre les expressions inusitées dans d'autres contextes. "Dans l'étude des mots, dit M. Bally, il faut non seulement savoir attendre, il faut savoir oublier ... La connaissance d'une langue est une habitude qui ne peut s'acquérir que peu à peu, et les tâtonnements sont la rançon d'une science solide et complète."'k On saisira mieux le jeu libre des mots en écoutant qu'en prenant part à une conversation. On doit apprendre les mots par groupes, par synonymes, et surtout, on ne saurait assez le dire, se défier des groupements étymologiques qui conduisent à des impropriétés d'expression.

Tout l'effort de M. Bally est dirigé contre les méthodes d'enseignement actuellement en usage. Il laisse entrevoir que la méthode historique ne vaut rien à l'école et qu'il faut suivre une méthode descriptive qui s'attache à l'étude de la langue parlée comme fonction de la vie, alors que jusqu'ici on étudiait la langue littéraire. Mais celle-ci n'est pas spontanée, seule la langue parlée peut élever la prétention de l'être. M. Bally constate que c'est

"4 E. Lerch, Litcralurblall f. girman. und roman. Philologir 1915, p. 125.

"L' L'tude systêmatique des moyens d'experssion, p. 13.

"Prócis de Stylislique, p. 37. 
le langage naturel, celui sur lequel devraient porter toutes les études stylistiques, qui est appris le plus mal à l'école. ${ }^{27}$ La langue parlée passant pour banale et vulgaire n'y est pas enseignée. Pour perfectionner la langue des élèves, il faudrait comprendre leur manière de parler. Pourquoi la pédagogie néglige-t-clle ce point de vue? C'est que l'enseignement est trop formaliste et trop intellectualiste, c'est-à-dire qu'il porte d'une part sur les formes extéricures du langage sans prendre garde à leur signification, d'autre part on essaye d'expliquer les matières du langage par les procédés de la logique. Or il s'agit de saisir les concepts fondamentaux, les formes habituelles de la pensée, voir ensuite comment ils s'associent entre eux et on s'efforcera de découvrir comment le système de notre pensée se reflète dans le système du langage.

M. Bally caractérise le préjugé littéraire, qui consiste à forcer les élèves de créer l'émotion littéraire. "Nous partons de l'idée, dit-il, que nos ćlèves doivent avoir de l'imagination, une sensibilité délicate et surtout du style." ${ }^{28}$ Mais sur une classe de vingt élèves, on trouverait à peine un tempérament littéraire. En cherchant à détourner les enfants des voies ordinaires du langage, on n'arrivera qu'à leur donner le goût du bizarre et ils seront incapables de dire simplement et nettement ce qu'ils pensent. On doit substituer à un idéal exclusivement littérairc l'étude générale de l'expression, et en accentuant ainsi le rôle du sentiment dans le langage, on ouvrira la voie à l'étude de la stylistique. ${ }^{29}$

Pour l'enscignement des langues on trouvera au commencement du Traitê de Stylistique un grand nombre d'idées fécondes. Ainsi la constatation que le langage exprime nos sentiments, si elle n'est pas nouvelle, est du moins formulée d'une façon très originale; de même l'idlée d'enseigner les langues modernes par la langue parlée avant d'aborder les textes écrits. On peut retrouver ces mêmes principes dans le livre de R. Hildebrand: Vom deutschen Unterricht, ainsi que dans le mouvement dit "réformiste," inauguré par Viëtor sous la formule "Der Sprachunterricht muss umkehren!" On a signalé qu'en 1887 déjà le professeur Schröder écrivait dans son opuscule Vom papicrnen Stil: "Unsere neuhochdeutsche Muttersprache, die Sprache unserer Denker und Dichter, ist ja nicht eine

${ }^{27}$ Stylistique et enseignement secondairc, p. $8 \mathrm{~s}$.

${ }^{28}$ Stylistique at enseignment serondaire, p. 13.

29 Ibid., p. 15. 
künstliche, sondern eine lebendige Sprache. Und Leben ist Geschmeidigkeit, Freiheit, innerer untrennbarer Zusammenhang, nicht blos mechanische Beweglichkeit säuberlich zerlegter Glieder."

Pour aborder les études stylistiques, M. Bally voudrait que l'on s'en tint à la langue maternelle, parce que la correspondance entre la parole et la pensée s'y manifeste de la façon la plus claire et la plus évidente. En partant de la langue maternelle on étudiera les langues étrangères des peuples civilisés, parce que ces langues tendant à se rapprocher dans leur lente évolution, forment une mentalité que M. Bally appelle la mentalité européenne. Dans l'étude de la langue maternelle M. Bally insiste sur la comparaison. Les normes de comparaison seront 1 . le mode d'expression intellectuel, auquel on mesurera les différences de sens entre les faits d'expression proprement dits et les éléments inaffectifs de la langue et 2. la langue commune usuelle, qui fera apparaitre par contraste les caractères affectifs produits par les faits d'évocation de milieu, c'est-à-dire les particularités sociales du langage. ${ }^{30}$

Pour résumer ces quelques pages nous dirons que la science que M. Bally nomme stylistique s'éloigne en beaucoup de points de la stylistique telle qu'on l'entend de coutume, c'est-à-dire qu'elle ne vise pas à l'étude des faits de langue du point de vue esthétique, mais qu'elle les ramène à la langue parlée pour en fixer par comparaison le degré d'affectivité. La langue usuelle est ainsi le critère de tout fait linguistique. Comme l'enseignement en a jusqu'ici tenu peu de compte, s'ingéniant à faire trouver aux élèves des effets de style, ceux-ci ne sauraient ni s'exprimer clairement et simplement, ni apprécier de juste façon un fait littéraire. La traduction ne doit plus rester au premier plan dans l'enseignement: on recourra à la description du fait de langage étudié, en réagissant contre l'instinct étymologique qui consiste à voir entre les faits de langue des relations étymologiques qui ne sont plus senties par les sujets parlants. Ce qui rend si utile la recherche des éléments intellectuels et affectifs d'un fait de langue, c'est qu'elle renouvelle la synonymie, car l'étude d'un état de langue n'est pas autre chose qu'une vaste étude de synonymie, puisque tout y est comparaison et opposition.

\section{University of Pennsylvania}

${ }^{30}$ Traile, p. $28 \mathrm{~s}$. 